\author{
IOANNIS PETROPOULOS \\ Università Democrito Di Tracia
}

\title{
PERCHÉ I MEDICI PARLANO ANCORA IL GRECO? ${ }^{1}$
}

\begin{abstract}
Although the extraordinary progress in medicine since the $19^{\text {th }}$ century has made Hippocrates and Galen irrelevant, Greek and Greek-derived terms continue to be used in the medical sciences today. The marked facility of the Greek language for word-formation facilitated the expansion of its medical lexicon. Greek medicine evolved far longer than its modern counterpart; its enduring cachet has lent it an atemporality. This article traces the main stages in the history of the nearly continuous reception of Greek medical nomenclature across more than two millennia. The process is shown to have been inseparable from the transmission and editing of Greek medical texts and their translation into Latin, Arabic, and eventually into vernacular languages. The article also sheds incidental light on the history of translation and transliteration in Europe and the Arab world.
\end{abstract}

\footnotetext{
1 Vorrei ringraziare di tutto cuore la Professoressa Athina Georganta (Dipartimento dei Beni Culturali, Universitá degli Studi di Padova), la quale ha inspirato il tema del presente articolo e ha incoraggiato in un modo particolare la mia ricerca scientifica per quanto riguarda il tema anticipato. Inoltre ringrazio la Professoressa Alessandra Coppola (dello stesso Dipartimento) che mi ha invitato a presentare il 06.05.2019, sotto il patrocinio del Centro Interdipartimentale di Ricerca sulla Storia dell'Aristotelismo e della Tradizione Classica, fondato di recente, il mio discorso su cui è basato il presente articolo. Vorrei anche ringraziare calorosamente la Dottoressa Rossella Di Schiadi per aver tradotto in italiano il presente articolo. Inoltre ringrazio il Professore Asimakis Fliatouras (Dimokritos Università di Tracia) dei suggerimenti bibliografici che hanno a che fare con la linguistica di termini tecnici moderni.
} 
KEY WORDS: Ancient Greek medicine, nomenclature, reception of ancient Greek medicine, textual transmission, translation, vernacular languages

Chirurgo, anestetico, cranio, diabete, flebite, bronchite, laringe, esofago, cachessia, arteria, sindrome, paraplegia. Sono tutti termini usati quotidianamente dai medici su scala internazionale. Alcuni termini tecnici, soprattutto sostantivi ed aggettivi, rientrano nella parlata quotidiana anche dei non specialisti, come: allergia, diarrea, nausea, terapia, trauma, dieta, amnesia, coma, sintomo, isterico, flemmatico, farmaceutico, ecc. Tutti i termini appena citati derivano dall'antica lingua greca. É, tuttavia, una questione complessa stabilire in che modo essi siano stati adottati. Durante questo mio audace tentativo di approcciare l'argomento in questione, ripercorrerò la storia dell'assunzione dei termini, nonché e soprattutto, della traduzione. La tematica da me trattata ben si adatta qui, cioè all'Università di Padova, poiché è proprio presso la Facoltà di Medicina del presente Ateneo, famosa da secoli, che compì i propri studi, dal 1794 al 1797, Ioannis Kapodistrias, il futuro Primo Governatore della Grecia, il capo di stato più importante della Grecia moderna. Egli, dopo essersi laureato a Padova all'età di ventun'anni, esercitò per alcuni anni la professione medica nella sua isola natale, Corfù.

Come accade oggigiorno con tutta la terminologia tecnica, anche quella medica dell' antichità era una lingua, se non un sottolinguaggio, la quale veniva usata da una minoranza di specialisti. Tucidide riconosce, senza ombra di dubbio, l'esistenza di una lingua squisitamente medica, quando, in occasione della descrizione della famosa epidemia scatenatasi ad Atene durante il secondo anno della guerra del Peloponneso, riporta, nel secondo libro della sua istoria, che 'seguirono tutte le forme di scarica biliare definite dai medici'. ${ }^{2}$ In questo specifico passaggio Tucidide, come nota ad locum Sir Kenneth Dover, si servì di un lessico come

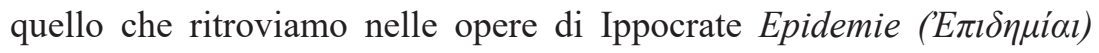

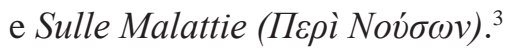

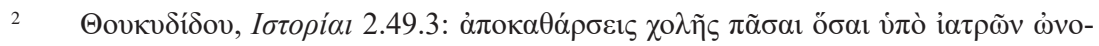

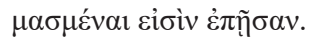

3 Dover 1997: 114. 


\section{L’ANTICHITÀ}

La lingua greca dell'antichità disponeva di un'ampia gamma di lingue tecniche come quella amministrativa, costruttivo-architettonica e filosofica, e quella che era collegata con l'allevamento di cani e con l'ippica. ${ }^{4}$ Torniamo però alla lingua medica. I termini medici dell'antichità (che del resto costituiscono, in gran parte, il modello di partenza della futura terminologia tecnica internazionale) sono particolarmente descrittivi. Definiscono 1) la struttura corporea, ovvero l'anatomia (termine greco), 2) il funzionamento dell'organismo (termine greco), 3) il sintomo o la patologia (termini greci), 4) alcune procedure mediche, e 5) la materia medica. ${ }^{5}$ Ho già citato due delle opere attribuite ad Ippocrate. Quel medico greco, chiamato, Padre della Medicina Occidentale, vissuto intorno al 460-370 a.C., era un contemporaneo di Socrate. A lui sono state attribuite ben 58 opere scientifiche da far risalire al V-IV secolo a. C., di natura eterogenea, divise in 73 libri, ed ognuna contraddistinta da uno stile letterario diverso. Questi libri, nel loro insieme, costituiscono il Corpus Hippocraticum. ${ }^{6}$ Egli vi patrocinava l'applicazione del pensiero razionale nel trattamento delle malattie, sostenendo, soprattutto, che le malattie erano provocate da cause naturali.

La medicina greca, e principalmente quella ippocratica, era stata introdotta a Roma sin dall'inizio del 200 a. C. e probabilmente, da molto prima, se si considera che il culto di Asclepio si introdusse a Roma nel 292 a. C., partendo da Epidauro. ${ }^{7}$ A partire dal III secolo a. C. e durante l'Impero, la maggioranza dei medici nel mondo romano erano greci o almeno portavano nomi greci, come rivelano le epigrafi funebri fino al III secolo d. C. ${ }^{8}$ Alcuni di loro erano schiavi o liberti. Intorno al 200 a. C. Catone il Censore accusò i medici greci immigrati di essere ciarlatani

\footnotetext{
$4 \quad$ Dover 1997: 112-117.

5 Cfr. Nagy 2013: 179-182.

$6 \quad$ Vedi Jouanna 1992 generalmente per Ippocrate. I medici egiziani di lingua greca durante i periodi ellenistico e romano inventarono termini anatomici in greco: vedi Jouanna 2012a: 19-20. Per la storia testuale del corpus, vedi lo stesso 2012b: 38-62. Vedi Lane Fox 2020 su una nuova esaminazione della nascita della medicina Ippocratica e del Corpus Hippocraticum.
}

$7 \quad$ Ferngren \& Amundsen 2005: 3841.

$8 \quad$ Vedi Nutton 2012: 535-536. 
criminali, sebbene egli, in una ricetta medica, menzioni come ingrediente il cumino nero servendosi del suo nome greco smyrnaeum e citi per medici, che considerata l'epoca, certamente dovevano essere greci. ${ }^{9}$

Il greco era la lingua della medicina ed arricchiva di termini tecnici quella latina. Catone osservava, non senza una certa ironia (secondo Plinio il Vecchio), che bastava scrivere in greco un'opera di contenuto medico per conferirle prestigio. La terminologia medica latina si plasmò, in gran parte, sui modelli greci, poiché la scienza medica latina non si era ancora sufficientemente sviluppata. Non vi era, infatti, un Ippocrate o un Galeno per poter disporre di concetti medici corrispettivi e di una corrispettiva terminologia unitaria. Dall'epoca d'Ippocrate fino a quella di Galeno (150 a. C. circa), la lingua greca continuò a sviluppare, per oltre 600 anni, il lessico medico. La terminologia medica greca si normalizzò sotto l'influsso di Galeno, il quale scrisse un'opera esprimendo l'esigenza di disporre di termini specifici. L'opera in questione, Sulla

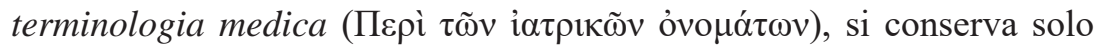
nella sua traduzione araba. ${ }^{10}$

A Roma, il processo di assimilazione della terminologia greca, ${ }^{11}$ ebbe una continuità che culminò in due periodi diversi: 1) Agli inizi del I secolo d. C., cioè all'epoca di Cornelio Celso, l'autore dell'enciclopedia medica, De Medicina; e 2) verso la fine del IV secolo d. C. fino al $\mathrm{V}$ secolo, quando, cioè, i medici autori come Marcello Empirico e 1'africano romano Cassio Felice raggiunsero il loro apice. Il fenomeno dell'assimilazione della lingua medica greca è trattato, in modo vario, da Celso ed altri autori. Si sviluppò attraverso 1) la traslitterazione e 1'adattamento del prestito alla morfologia della lingua latina (ad esempio, stomachus, herpes, glaucoma), 2) attraverso i calchi, dai quali derivano nuove forme nella lingua destinataria (ad esempio, dentes canini è un calco della parola greca kynodontes), 3) attraverso la traduzione letteraria o prestito semiologico, dove una parola nella lingua destinataria adotta un nuovo significato. Per esempio, il lemma musculus (Celso 5.26.3), diminutivo di mus, rende il termine greco mus che per Ippocrate ed Aristotele possiede un significato metaforico e medico, così

\footnotetext{
$9 \quad$ Plinio il Vecchio, Naturalis Historia 29.7.17 ff.; Henrichs 1995: 248-249.

$10 \quad$ Mayer 1946: 403 n. 18; Montero-Cartelle 1997-1998: 230 n. 7. Su la terminologia di Galeno, vedi Durling 1993 e Boudon-Millot 2012.

11 Su questa assimilazione, cfr. Montero-Cartello 1997-1998: 227-248.
} 
come cancer è la traduzione letterale del termine ippocratico karkinos (granchio), 4) ed infine, attraverso la glossa, ovvero la spiegazione perifrastica di un termine. Celso ed altri autori di medicina, ricorrono a frasi stereotipate, a formule, spesso introducendo un termine straniero, greco. Queste frasi sono: 'quello che in greco si chiama...ciò che i greci chiamano....ciò che viene definito dai greci.' Leggiamo: 'ascesso ...che i greci chiamano apostemata' (Celso 2.1.1), 'la tosse umida che i greci chiamano ygrobecha'(Cassio Felice 34.1), 'bagno essiccativo, che viene chiamato dai greci xerolusia' (Cassio Felice 76.14). ${ }^{12}$ Celso, definito dal suo contemporaneo Columella 'il Cicerone della medicina', che non era un medico ma un enciclopedista eclettico, non sviluppò un lessico tecnico. Nonostante fosse uno strenuo difensore della latinitas, almeno il $26 \%$ dei termini medici da lui usati hanno origine greca. ${ }^{13}$ Il fatto che a partire del III secolo il 45\% dei termini tecnici presenti nelle opere di Prisciano e Cassio Felice erano greci, dimostra la sempre più evidente assimilazione degli ellenismi da parte della medicina romana. ${ }^{14}$

\section{LA TARDA ANTICHITÀ E LE TRADUZIONI IN ARABO NEL PERIODO MEDIEVALE}

Per quanto riguarda l'Oriente, il quadro della storia medica cambia. Innanzitutto, nel V secolo d. C. ad Alessandria d'Egitto fu raccolto un insieme di testi attribuiti ad Ippocrate ed a Galeno. Questo corpus getta le fondamenta dell'educazione medica a Bisanzio ed a Ravenna. La teoria e, in parte, la terminologia medica greche, si diffusero in Occidente, durante il Medioevo tardo, proprio grazie alle traduzioni in arabo di Ippocrate, Galeno ed altri medici greci. Dal XI secolo in poi, i medici studiavano Galeno da traduzioni latine tratte da quelle in arabo. Ma in che modo furono trasmesse le traduzioni in arabo?

Alla metà dell'VIII secolo a Baghdad, durante il regno della dinastia degli Abbasidi, nacque un movimento di traduzione dall'arabo dei

\footnotetext{
12 Per la terminologia di Cassio Felice vedi Fraisse 2006: 147-158; per la terminologia usata per Marcellino Empirico (fine del IV secolo/inizio del V secolo), vedi Jouanna-Bouchet 2009: 720-741.

13 Montero-Cartelle 1997-1998: 229-230 n. 6.

14 Montero-Cartelle 1997-1998: 229-230 n. 6.
} 
testi più importanti non solo della medicina greca, ma anche della filosofia e delle scienze naturali. ${ }^{15}$ Le traduzioni divennero sistematiche e aumentarono sotto l'egemonia del califfo al-Mamun (che regnò nel inizio del IX secolo: 813-833). Secondo una leggenda, il suddetto califfo inviò eruditi arabi insieme a un traduttore a Costantinopoli affinché raccogliessero dei manoscritti. ${ }^{16}$ Le traduzioni furono sovvenzionate da membri dell'elite e dai califfi Abbasidi. Tra coloro che sovvenzionarono le traduzioni vi erano i membri di una famiglia di medici cristiani grecofoni di nome Bukhtishu.

Molte opere antiche furono tradotte in arabo direttamente dalla lingua greca, mentre altre furono tradotte attraverso una traduzione siriana o ebraica, oppure attraverso la traduzione ebraica di una siriana. ${ }^{17}$ Questo significa che quando, come vedremo, negli anni che seguirono furono tradotte le opere di autori greci dall'arabo in latino, le traduzioni che ne scaturirono si allontanavano di 4 o 5 gradi dalle versioni originali. Già verso la fine del IX secolo, i medici arabi seguivano le tracce di Ippocrate e di Galeno sugli umori. Il traduttore per eccellenza di Galeno era il cristiano nestoriano Hunayn ibn Ishaq (morto intorno all'873), noto in Occidente come Ioannizio.

Il filosofo e medico persiano Ibn Sina, noto a noi come Avicenna, fu il rappresentante più importante dell'epoca d'oro della scienza medica araba (IX-XIII secolo). Egli morì nel 1037. Nella sua enorme opera composita, Il Canone della Medicina, scritto prima del 1015, Avicenna sistematizza, secondo i criteri della logica aristotelica, le teorie mediche di Galeno; e incluse elementi della medicina da Egitto faraonica e Sumeria. Intorno al 1150 e forse più tardi (1150-1187), il Canone fu tradotto in latino da Gherardo da Cremona, uno tra i più prolifici traduttori nella storia dell'Europa. Apparteneva alla cosiddetta Scuola di Traduzione di Toledo, allora rinomata per il clima cosmopolita e di tolleranza religiosa. ${ }^{18}$ Il Canone, che codificò una gran parte di medicina unani, cioè

15 Vedi Savage-Smith 2013, in particolare 141-151 sulle prime traduzioni in arabo e sulla medicina medievale dell'Islam. Inoltre, vedi Anawati 1977: 765-779.

16 Mattock 1992: 83.

17 In generale vedi Tolan et al. 2012: 87-93. Per il lavoro di traduzione degli Ebrei durante il Medioevo nel Mediterraneo, vedi Ferre 2012: 522-562.

18 Su Gherardo e la Scuola di Traduzione di Toledo, vedi Arráez-Aybar et al. 2015, in particolare 23-25 e Burnett 2001: 249-288. 
greca antica, fu incluso nel curriculum delle università in Samarcanda e Tashkent, e a partire dal XIII secolo, in India.

Già a partire dallo stesso secolo, il Canone, tradotto da Gherardo, divenne, presso l'Università di Bologna, un manuale fondamentale. Da allora e fino alla fine del XVIII secolo, il testo in questione, spesso revisionato, divenne il Vangelo della medicina, per esempio a Oxford. Il Canone fu ristampato più di quaranta volte in Europa. A Padova fu stampato per la prima volta nel 1476. Fino al XVI secolo, l'opera di Avicenna costituì una cospicua fonte di termini di anatomia, molti dei quali furono translitterati dal greco. Ad esempio, il termine greco ' $\delta$ i $\alpha \rho \rho \alpha \gamma \mu \alpha$ ' (diafragma) fu da lui reso diafragma. Questo ultimo fu, anche, il termine adottato da Gherardo. Andrea Alpago, originario di Belluno, professore di medicina a Padova nel 1522 (una cattedra occupata per soli due mesi a causa della sua morte), curò e commentò la traduzione di Il Canone di Gherardo. Conosceva l'arabo perché era vissuto a Damasco. ${ }^{19}$

\section{RINASCIMENTO}

Passiamo ora al Rinascimento. A partire dal XIII secolo, grazie alla trasmissione delle traduzioni latine come quelle della Scuola di Toledo, il pensiero greco (specialmente Aristotele e la scienza medica), giunsero nell'Europa latina 'indossando il turbante' ${ }^{20}$ ed imprimendo grandi cambiamenti nel campo dell'istruzione e dell'intelletto. Ciononostante, per gran parte del Medioevo, soltanto quaranta delle mille traduzioni di opere mediche furono tradotte in latino. Dal 1350 al 1600 si moltiplicò notevolmente il numero dei manoscritti di autori greci antichi. ${ }^{21}$ Letterati occidentali in visita a Bisanzio ed in seguito letterati bizantini immigrati, portarono con sé questi manoscritti, i quali oggi compongono gran parte della letteratura antica greca salvata. Questi testi (tra cui anche testi medici), furono tradotti in latino e talvolta commentati da umanisti del XV del XVI secolo.

\footnotetext{
19 Su Alpago vedi Lucchetta 1964.

20 Tolan et al. 2012: 92.

21 Per le edizioni e la diffusione degli antichi scrittori medici greci, vedi Nutton 2002: 461-478 e Jackson 2012: 378-390.
} 
Un interesse generale per le traduzioni precise in latino di testi della letteratura antica greca fu rafforzato da due letterati greci residenti in Italia: Emmanuele Crisolora (1355-1415) e Costantino Lascaris (14341501), i quali sancirono la traduzione quale principio dell'educazione umanistica. ${ }^{22}$ Una spinta ulteriore a questo movimento di traduzione fu data da Demetrio Calcondila, professore di lettere greche a Padova dal 1463 al 1472. Chalkokondili (Calcondila) tradusse in latino il trattato di

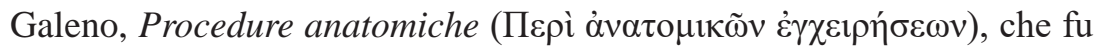
pubblicato dopo sua morte (1531).

Durante il Rinascimento, le già esistenti traduzioni latine vennero corrette ed in un secondo momento, ad esse si aggiunsero nuove traduzioni latine, attenendosi, questa volta, a testi originali d'Ippocrate e Galeno. ${ }^{23} \mathrm{Sin}$ dal XVI secolo, letterati avevano tentato di ripulire il linguaggio medico dai termini arabi. Durante questo periodo, gli intellettuali erano alla ricerca del ritorno ad fontes, ovvero ai testi originali. I letterati, potendo ormai accedere ad edizioni stampate delle opere originali, criticavano gli errori, talvolta anche ingiustamente, che trovavano nelle traduzioni medievali arabo-latine. ${ }^{24}$

Colui che contribuì alla svalutazione della medicina araba di Avicenna ed alla consolidazione d'Ippocrate e Galeno, fu Niccolò Leoniceno (1428-1524), professore di filosofia e medicina presso Padova e Ferrara. ${ }^{25} \mathrm{Fu}$ il traduttore più rilevante dei due suddetti medici greci, durante il Rinascimento. Tradusse undici opere di Galeno. Possedeva, inoltre, la collezione più ampia di testi antichi filosofici e medici del mondo. La ripulitura del linguaggio medico dai termini arabi avenne gradualmente e si concluse solo dopo la pubblicazione, nel 1555, della seconda e migliorata edizione di De humani corporis fabrica del padre dell'anatomia Andrea Vesalio, professore di medicina a Padova. ${ }^{26}$ Alcuni, come il letterato veneziano Giorgio Valla, si servivano della terminologia estratta dal dizionario atticista del secolo II d. C. del contemporaneo di Galeno

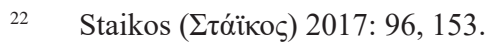

23 Durling 1961, in particolare 236s.; Jackson 2012: 378-390; Nutton 2002: 461478; Bacalexi 2009: 201-222 e Bacalexi 2012: 89-106.

$24 \quad$ Durling 1961: 238.

25 Nutton 1997: 2-19.

26 Russell 2013, in particolare 1-14. 
Polluce, l'Onomasticon, i cui manoscritti furono scoperti alla fine del $\mathrm{XV}$ secolo. Nel quarto libro sono riportati termini medici.

La trasmissione della medicina greca è dovuta alle pubblicazioni di Manuzio, le quali, alla sua morte nel 1515, furono curate dai suoi successori. Furono usati manoscritti di questi autori, manoscritti che si trovavano in Italia. L'edizione aldina di Galeno in cinque tomi (15251526) ed in seguito quella di Ippocrate (1526), erano alla base di tutte le seguenti edizioni e traduzioni dei suddetti autori. ${ }^{27}$ Nel XVI secolo si riscontrò un'esplosione di traduzioni latine di Ippocrate e di Galeno, specialmente a partire dagli anni 40 del 1500. Più di 630 opere tradotte furono pubblicate a Parigi, Lione, Venezia e Basilea. Dal 1546, il mercato studentesco fu inondato di traduzioni tascabili a Parigi e particolarmente a Lione. Nello stesso periodo, d'altra parte, furono pubblicate sporadiche traduzioni di Galeno e di altri medici greci in francese, inglese ed italiano.

L'abbondanza di traduzione - un autentico mercato aperto - permise ai lettori di metterle a confronto. I traduttori, ormai, estraevano termini tecnici da Celso (che ho già citato); sua enciclopedia fu pubblicata per la prima volta a Firenze nel 1478 e era molto popolare fino a XIX secolo. Ma non bastava la lingua latina e per questo sono stati conservati i termini originali, spesso latinizzati. 'Come si potrebbe rendere il termine pathos?' si chiedeva Thomas Linacre, il collaboratore oxfordiano di Manuzio. I termini composti dehydratio, haemolysis, dyspnoe, furono inventati ed adottati durante il cinquecento.

Tutti quelli che traducevano antiche opere di medicina nella lingua vernacolare durante il Rinascimento era inevitabile di ricorrere ai termini greci (traslitterati) e latini, dato che questa terminologia da una parte trasudava il prestigio dell'antica tradizione e perché dall'altra parte le lingue parlate (vernacolari), essendo in fase di sviluppo e fluidità, non avevano ancora un vocabolario scientifico sufficientemente equivalente. L'uso dei termini degli antichi auctores, inoltre, preveniva la possibilità di fraintendimenti e confusioni. Tuttavia, nel XVI secolo alcuni medici cercarono spesso di colmare questo vuoto nei loro scritti facendo riferimento accanto ai termini antichi corrispondenti termini in francese o in italiano. Il ricorso al "bilinguismo" - cioè la coesistenza di termini

$27 \quad$ Nutton 2002: 461-462. 
antichi in parallelo con termini moderni - si preferiva perché gli autori non conoscevano il livello della conoscenza dell'antichità dei loro lettori. I termini moderni furono gradualmente consolidati durante questo periodo. $^{28}$

\section{DAL XIX SECOLO AD OGGI}

Dunque, per motivi di carattere storico, sin dall'epoca di Catone, la lingua greca antica divenne la lingua di prestigio della scienza medica. La longeva supremazia scientifica della medicina greca fu confermata dall'osservazione di Orazio: La Grecia conquistata, conquistò il ferro vincitore. ${ }^{29}$ Nella storia dell'assunzione della terminologia, il ruolo principale fu assegnato alla translatio (per usare il termine latino), con una duplice funzione: da un canto, quella dello spostamento dei manoscritti e dall'altro quella della traduzione (linguistica). Le origini della translatio linguistica si rintracciano essenzialmente a Baghdad nel IX secolo, nelle traduzioni arabe. Un ruolo determinante nella trasmissione e traduzione dei testi dei medici greci, ebbero la Scuola di Traduzione di Toledo fino a metà del XIII secolo, e le università italiane, soprattutto nel '400 e nel '500. A Baghdad come in Spagna ed Italia, lo studio e la traduzione dei testi greci scaturiva dalla ricerca della sapienza greca.

Sebbene questo articolo si occupi principalmente della storia precedente della terminologia medica, va menzionato il XIX secolo, che fu il periodo più decisivo per la creazione e la perpetuazione della nomenclatura medica greca fino ad oggi. L'evolversi della scienza medica, soprattutto a partire dal XIX secolo, impose la necessità di arricchire la terminologia ad essa relativa. ${ }^{30}$ La tradizione della terminologia greca e latina - era ed è ancora oggi così potente che la lingua greca è divenuta la matrice produttrice di numerosi neologismi con radice greca come enzima, microbo, cromosomo, metabolismo ed una pleiade di termini con la desinenza -ologia (come l'ibrido otorinolaringologia). Altri termini

28 Per l'emergere delle lingue nazionali, soprattutto in relazione alla scrittura di opere mediche del XVI secolo e alle soglie della storia moderna, vedi ad esempio Siraisi 1990; Jacquart 1997; Montagne 2017.

29 Epistulae 2,156.

30 Banay 1948: 1-27; Jóskowska, Grabarczyk 2013: 41-52. 
nuovi sono scaturiti dall'uso di termini antichi con un nuovo significato (tifo, metastasi), e dalla produzione di parole composte greco-latine come cardiovascolare. In linee generali, si può dire che nelle lingue come l'inglese e l'italiano, i termini relativi alla fisiologia ed alla patologia del corpo, hanno origini greche. La maggior parte degli termini anatomici è latina. Il vantaggio delle terminologie greca e latina e di quella ibrida greco-latina sta nel fatto che sono chiare e, si direbbe, quasi trasparenti, pur conservando una neutralità emotiva.

Come accade con il mito antico greco, così anche la lingua d'Ippocrate e di Galeno è divenuta atemporale, trascendendo il corpo umano e conservando il prestigio e la chiarezza descrittiva. La medicina greca, così come la scienza greca più in generale, ebbe una durata maggiore rispetto a quella moderna. I termini derivati dal greco e latino sono usati, nonostante il fatto che la moderna scienza medica non sia basata su Ippocrate e sui suoi successori. Durante la cerimonia di assegnazione del premio Nobel per la Letteratura, il poeta Odisseas Elitis notò che, in quasi trenta secoli dall'età di Omero, non è esistito secolo in cui non si sia scritta poesia in greco. Lo stesso vale per la medicina. Circa dall'anno 450 a. C. e quasi senza interruzione, la lingua greca viene usata in medicina e non solo dai grecofoni.

\section{BIBLIOGRAFIA}

Anawati G., 1977, 'Science', [in:] Cambridge History of Islam, P. M. Holt et al. (edd.), Cambridge, pp. 741-779. https://doi.org/10.1017/CHOL9780521219495.015.

Arráez-Aybar L.-A., Bueno-López J.-L., Raio N., 2015, 'Toledo School of Translators and Their Influence on Anatomical Terminology', Annals of Anatomy 198, pp. 21-33. https://doi.org/10.1016/j.aanat.2014.12.003.

Bacalexi D., 2009, 'Trois traducteurs de Galien au XVIe siècle et leur regard sur la tradition arabe', [in:] Pratique et pensées médicales à la Renaissance: Actes du 5le colloque international d'études humanistes (Tours, 2-6 juillet 2007), J. Vons (ed.), Paris, pp. 201-222.

Bacalexi D., 2012, 'Galien, d'une réception à l' autre: Tradition médiévale arabe et humanistes du XVIe siècle', Seizième Siècle 8, pp. 89-106. https://doi. org/10.3406/xvi.2012.1045. 
Banay G. L., 1948, 'An Introduction to Medical Terminology I. Greek and Latin Derivations', Bulletin of the Medical Library Association 36/1, pp. 1-27.

Boudon-Millot V., 2012, Galien de Pergame: Un médecin grec à Rome, Paris.

Burnett C., 2001, 'The Coherence of the Arabic-Latin Translation Program in Toledo in the Twelfth Century', Science in Context 14/1-2, pp. 249-288, https:// doi.org/10.1017/S0269889701000096.

Dover K. J., 1997, The Evolution of Greek Prose Style, Oxford.

Durling R. J., 1961, 'A Chronological Census of Renaissance Editions and Translations of Galen', Journal of the Warburg and Courtauld Institutes 24/3-4, pp. 230-305. https://doi.org/10.2307/750797.

Durling R. J., 1993, A Dictionary of Medical Terms in Galen, Leiden-New York, NY. https://doi.org/10.1163/9789004377301.

Ferngren G. B., Amundsen, 2005, 'Aesclepius', [in:] Encyclopedia of Religion, vol. VI/2. ed., L. Jones (ed.), Detroit, MI, p. 3841.

Ferre L., 2012, 'The Jewish Contribution to the Transmission of the Cultural Legacy', European Review 20/4, pp. 522-562. https://doi.org/10.1017/ S1062798712000129.

Fraisse A., 2006, 'Néologismes et premières attestations dans le "De medicina" de Cassius Felix', Latomus 65/1, pp. 147-158.

Henrichs A., 1995, 'Graecia Capta: Roman Views of Greek Culture', Harvard Studies in Classical Philology 97, pp. 243-261. https://doi.org/10.2307/311309.

Jackson D. F., 2012, 'Greek Medicine in the Fifteenth Century', Early Science and Medicine 17, pp. 378-390. https://doi.org/10.1163/1573382320120002.

Jacquart D., 1997, Lexique de la langue scientifique: Astrologie, mathématiques, médecine, Paris.

Jouanna J., 1992, Hippocrate, Paris.

Jouanna J., 2012a, Greek Medicine from Hippocrates to Galen. Selected Papers, transl. N. Allies, Leiden- Boston, https://doi.org/10.1163/9789004232549.

Jouanna J., 2012b, 'Textual History', [in:] The Cambridge Companion to Hippocrates, P. E. Pormann (ed.), Cambridge, pp. 38-62. https://doi. org/10.1017/9781107705784.004.

Jouanna-Bouchet J., 2009, 'Composition littéraire et composition médicale: Un exemple remarquable dans la littérature médicale latine: Marcellus Empiricus', Latomus 68/3, pp. 720-741.

Jóskowska K., Grabarczyk Z., 2013, 'Greek and Latin in Medical Terminology', Folia Medica Copernicana 1/2, pp. 41-52. 
Lane Fox R., 2020, The Invention of Medicine: A History from Homer to Hippocrates, New York.

Langslow D. R., 2000, Medical Latin in the Roman Empire, Oxford.

Lucchetta F., 1964, Il medico e filosofo Bellunese Andrea Alpago († 1522), traduttore di Avicenna. Profilo biografico, Padova.

Mattock J. N., 1992, 'Islam', [in:] Perceptions of the Ancient Greeks, K. J. Dover (ed.), Oxford-Cambridge, MA, pp. 79-99.

Mayer C. F., 1946, 'Arabism, Egypt, and Max Meyerhof', Bulletin of the History of Medicine 19, pp. 375-432.

Montagne V., 2017, Médecine et rhétorique à la Renaissance. Le cas du traité de peste en langue vernaculaire, Paris.

Montero-Cartelle E., 1997-1998, 'El lexico médico latino entre la antigüedad y el renacimiento: la asimilacion de modelos médicos y léxicos', Voces (Caen) 8-9, pp. 227-248.

Nagy K. I., 2013, 'The History, Peculiar Terminology and Translation Problems of the Language of Medicine', LDMD 1, pp. 179-189.

Nutton V., 1997, 'The Rise of Medical Humanism: Ferrara, 1464-1555', Renaissance Studies 11/1, pp. 2-19, https://doi.org/10.1111/j.1477-4658.1997. tb00008.x.

Nutton V., 2002, 'The Diffusion of Ancient Medicine in the Renaissance', Medicina nei secoli-Arte e scienza 14/2, pp. 461-478.

Nutton V., 2012, 'Galen and Roman Medicine: Or Can a Greek Become a Latin?', European Review 20/4, pp. 534-542. https://doi.org/10.1017/ S1062798712000105.

Russell G. A., 2013, 'Vesalius and the Emergence of Veridical Representation in Renaissance Anatomy', Progress in Brain Research 203, pp. 3-32. https://doi. org/10.1016/B978-0-444-62730-8.00001-3.

Savage-Smith E., 2013, 'Medicine in Medieval Islam', [in:] Cambridge History of Science, D. C. Lindberg, M. H. Shank (eds.), pp. 139-167. https://doi. org/10.1017/CHO9780511974007.007.

Siraisi N., 1990, Medieval and Early Renaissance Medicine. An Introduction to Knowledge and Practice, Chicago. https://doi.org/10.7208/ chicago/9780226761312.001.0001.

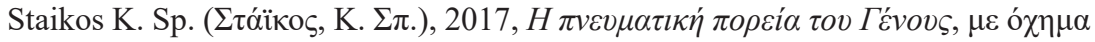

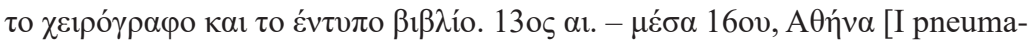
tiki poreia tou Genous, me ochima to cheirografo kai to entypo vivlio. 13os ai. - mesa 16ou, Athina]. 
Tolan J. et al., 2012, Europe and the Islamic World: A History, Princeton. https:// doi.org/10.23943/princeton/9780691147055.001.0001. 